\title{
Adaptive Path Selection using Meta-heuristic Ping Method
}

\author{
Simarpreet Kaur \\ PG Student, Dept. of ECE, \\ Shaheed Bhagat Singh \\ State Technical Campus, \\ Ferozepur(152001), India
}

\author{
Amit Grover \\ Assistant Professor, \\ Dept.of ECE, Shaheed Bhagat Singh \\ State Technical Campus, \\ Ferozepur(152001), India
}

\begin{abstract}
The unmanned aerial vehicles (UAV) can be used in various fields such as weather applications, military applications, remote sensing, oceanography etc. There are many problems for UAV to find best and obstacle free path by detecting obstacle and calculate turn. In this paper, a novel method has proposed model for the obstacle free UAV routing by using the multi-factor distances. The proposed model is entirely based upon the UAV turn calculation based shortest path with minimum number of obstacles and turns. The multi-factor distance has been computed which includes the distance between the source and destination, UAV and obstacles, obstacles and obstacles, angle of rotation (Degree of rotation) and other several factors. This proposed model is also sufficient to track the fuel and find best have been obtained in the form of various network parameters of latency of the hurdle detection. energy consumption, load, route persistence comparison. The experimental results have advocated the robustness of the path planning solution for the UAV path planning.
\end{abstract}

\section{Keywords}

UAV Routing, collision avoidance, alternative path planning, collision detection

\section{INTRODUCTION}

An pilotless aerial vehicle (UAV), usually termed as a vehicle without pilot. It is an aircraft which can fly without a human pilot aboard. UAV is famous with name called as drones. UAV can be controlled without a pilot by remotely or by installing $\mathrm{pc}$ in it. The fly of UAV can be controlled by a programmed framework or an outer administrator on the ground. Generally, UAVs were straightly fly by using remotely guided airplane, The Best UAV which can flew remotely in heartbeat plane is Nazi-German V-1, Its upgradation was the Nazi-German V-2

The best use of Unmanned aerial vehicles (UAVs) are is for military operations and considered for regular citizen applications, for example, natural observing [1]. For this best software must be required to troublesome optimization problems, for example, variations of the weapon-target task issue [2]. For the use of UAVs for observation and checking, choosing of advancement of the environment is neccessary, which create some type of control issues

As UAVs flew on given path so Path planning for small Unmanned Aerial Vehicles (UAVs) become biggest exploration now day. Little UAVs can be used for regular citizen applications, for example, fierce blaze administration [1], weather and hurricane monitoring [2], [3], and pollutant estimation [4]. Inspite of extensive UAVs, little UAVs are moderately easier to work and are fundamentally less expensive. Maintenance of fly of small UAV is easy because it fly at low altitude. Indeed, even in military applications, little vehicles [5] are mostly used because they fly at low altitude so easily handled by a person.

Despite of many advantages it still has disadvantages, like fuel refilling. As little UAVs flew by using fuel, it may not be workable for a UAV to finish an observation mission before refueling at one of the Station. For example, consider a mission where UAV have to fly from a point and reached to a target. During this mission it might be possible that fuel tank may get empty. Then UAV have to stop and look for the station for refueling. So it is to be accepted that UAV must be refueled to complete the mission. In this mission the main objective is over and over visit a few terminals keeping in mind the end goal to refuel again before going to every one of the objectives.

\section{LITERATURE REVIEW}

In [1]the author proposed work about vehicular ad hoc networks that falls under category of mobile ad hoc networks which provide communication between and among nearby vehicles.as the network is open so there is chance for security attacks so in this solution for this attacks is proposed which is known as DOS based attacks which adds a security to the existing solutions.In[2] the best solution in future to avoid major accidents the author introduce new vehicles which would be equipped with shortrange radios that are having the ability to communicate with the nearby vehicles and distance will be around one kilometer. The information that will be sent to a vehicular network will have the impact on life and death and add security to driving. However this approach is dependent on viable security solutions that will be accepted or not to goverenment or public. In[4]the serious issue of sybil attacks is discussed and a security scheme is also introduced for localizing and detecting the sybil attacks.For the sake of position verification accurately,two static algorithms are proposed. In[5] Vehicular sensor networks come up as a new applications that changed the scenerio of human driving and also controlled the traffic systems.In this a digital signature scheme is shown to be very effective for VSNs to carefully achieve the authentication.But because of presence of limited interval of $300 \mathrm{~ms}$ so efficient batch signature verification scheme is introduced which communicate between vehicle and RSUs. In[7] the most concerned problem in unmanned aerial vehicle is introduced that is target search.This problem is solved computationally.In this approach the optimal sensor performance is made for every candidate in the routing control problem. In [8], the authors had proposed an algorithms for routing an unmanned aerial vehicle in the presence of refueling depots. The objective of the problems to find a path for the UAV such that each target is visited at least once by the vehicle, the fuel constraint is rarely profaned on 
the trail for the UAV, and also the total fuel needed by the UAV may be a minimum. In [9] had planned the routing rule for 2 pilotless Aerial Vehicles with communication constraints.Contact with UGS is strictly maintained, that permits the UGS act as beacons for relative navigation eliminating the necessity for dead reckoning. This downside is brought up because the Communication strained UAV Routing downside (CCURP). to resolve the CCURP, shortest methods between targets ar computed by means that of a graph transformation. Given the shortest methods between targets, 2 resolution ways ar given .In [10], have worked on multi-Objective UAV routing methodology. the aim of the study, given during this paper, has been to use a multiobjective graph-based methodology for arrange routes of a simulated UAV taking under consideration situations with danger zones or prohibited areas, characteristic of civil airspace, similarly as areas with time and speed restrictions and a few negotiable "safe corridors". In [11], have worked on UAV routing protocols for distributed sensing element information exfiltration. the matter self-addressed during this paper is information exfiltration from a group of sensors that ar unable to determine ad-hoc communication owing to their widespread preparation, geographical constraints, and power issues. sensing element information is exfiltrated by one or additional unoccupied aerial vehicles (UAVs) that act as information mules by visiting every sensing element so as to determine a communication link.

\section{EXPERIMENTAL DESIGN (PROPOSED WORK)}

At first, we consider the active ping mechanism using the UAV path planning (UPP) algorithm to plan the UAV's path between the two points, which includes the double step evaluation. The first step consists of the take off position to the target position and other includes the target to the landing location. The path with hurdles or no hurdles is evaluated by using the multi-directional ping mechanism for the path evaluation. The probability of the availability of the hurdle goes up with the active ping agent. This is the probability $\mathrm{F}$ for the failure of the system:

$\mathrm{F}=(1-\mathrm{a}) 2$

Thus, the availability of the system, A, is

$\mathrm{A}=1-\mathrm{F}=1-(1-\mathrm{a}) 2$

\section{Algorithm 1: UAV path planning (UPP) Algorithm \\ UAV path planning algorithm initiates its operations for evaluating the existence of the hurdles on the way for continuous path updation.}

(1) UPP module initiates and establish the continuous path scanning procedure

(2) UPP module verifies the local UPP agent which is responsible for the ping discovery to detect the objects on the way

a) if UPP agent test verified successfullyUPP Agent is registered and executed.

b) OtherwiseUPP module returns the error and warns the base station about the failure occurred.

(3) If the step 2(a) is found true, do the following

a) Continuously check for the hurdles on the path by using the ping mechanism b) If ping is received back, then he hurdle recognition method boots up.

a) if hurdle is found

i. Run the distance evaluation and path updation program

b) Otherwise

i. Go to 2(a)

(4) If 3(b) return the true value, then do the following UPP agent will execute the distance evaluation program for the hurdle distance evaluation

a) $[$ dLoc timeToReach $]=f(x)\{n(x), X, Y, Z\}$; // where the hurdle lies and estimates the time to reach the hurdle

b) $\quad \operatorname{dLocN}\{\}=\mathrm{f}(\mathrm{x})\left\{\mathrm{n}(\mathrm{x}), \quad\left\{\mathrm{X}^{\mathrm{o}} \ldots \mathrm{X}^{\prime}\right\}\right\} ; \quad / /$ where the neighboring hurdles lie and estimate if they will approach in the path or not

c) $n$ Points $=\mathrm{f}(\mathrm{x})\left\{\left(\mathrm{n}(\mathrm{x}),\left\{\mathrm{X}^{\mathrm{o}} \ldots \mathrm{X}^{\prime}\right\}\right)+(\mathrm{n}(\mathrm{x})\right.$, $\left.\left.\left\{\mathrm{X}^{\mathrm{o}} \ldots \mathrm{X}^{\prime}\right\}\right)\right\}$; // where there is no hurdle detected in the ping area

(5) Calculate the distance of hurdle

(6) Calculate the nPoints with no hurdle and found the position with minimum degree of rotations

(7) If degree of rotation is less than the maximum angle i. Update the path with rotation angle

(8) Otherwise

i. Find the point of elevation from the nPoints array

ii. Return the degree of elevation

Update the path with elevation angle

In on-demand module, the route discovery procedure is used by nodes to obtain routes on an 'as needed' basis. In proposed UAV routing, route discovery works as follows. Whenever a UAV needs the route to a destination, it initiates a route discovery by flooding the ping signal to evaluate the hurdles over the path. When an intermediate hurdle is detected, the alternative path is evaluates to continue the flight towards the destination. The ping module uses the sonar signal for the hurdle detection up to the given range $(\mathrm{R})$. In addition, if there is a valid route available for the destination, the continuous path information is updated; otherwise, it re-broadcasts the ping till the hurdle is found or the destination is reached. The UPP on receiving the multiple ping hurdles and angular shifts are applied among the solution till the destination is evaluated continuously. As the ping proceeds towards the destination, it establishes the successful forward path to the destination by updating the path information at each point of hurdle. Route maintenance is done by means of number of ping hurdles and successful and failed attempts.

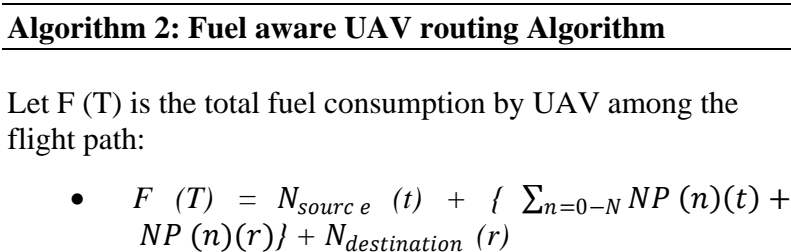


// where $\mathrm{t}$ is time of flight, $\mathrm{r}$ is the rotation angle, $\mathrm{N}$ gives not of initial segments, $\mathrm{N}_{\text {source }}$ is a takeoff position and $\mathrm{N}_{\text {destination }}$ gives the landing position.

- $\quad$ Suppose $\mathrm{F}\left(\mathrm{T}_{\mathrm{PN}}\right)$ is the Fuel consumption per segment among path (point to point straight flight)

- $\quad \mathrm{F}\left(\mathrm{T}_{\mathrm{PN}}\right)=\mathrm{EC} \sum_{\mathrm{i}=0 \ldots . \mathrm{j}}\left(\mathrm{N}_{0}(\mathrm{t}) * \mathrm{n}+\mathrm{N}_{0}(\mathrm{r}) *\right.$ $\mathrm{n}),\left(\mathrm{N}_{1}(\mathrm{t}) * \mathrm{n}+\mathrm{N}_{1}(\mathrm{r}) *\right.$ $\mathrm{n}), \ldots \ldots \ldots \ldots \ldots\left(\mathrm{N}_{\mathrm{j}}(\mathrm{t}) * \mathrm{n}+\mathrm{N}_{\mathrm{j}}(\mathrm{r}) * \mathrm{n}\right)$

// where $\mathrm{n}$ is the no. of path segments, $\mathrm{i}$ is the number of rotation or elevation angles, $\mathrm{E}\left(\mathrm{T}_{\mathrm{PN}}\right)$ depicts the straight distance between two points, between each point, $t$ depicts the per rotation angle energy, $r$ gives the per elevation angle energy

\section{RESULT ANALYSIS}

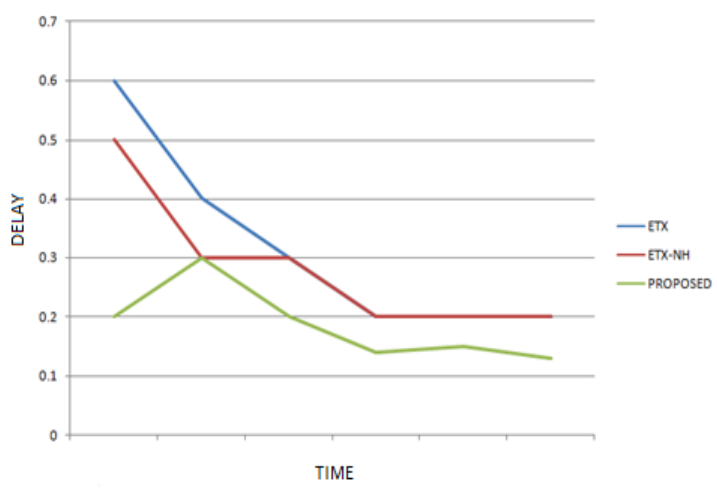

Fig 1: The comparison graph of latency of the hurdle detection

The comparative analysis depicts that the proposed algorithm performs much better than the existing algorithm. The proposed work is based upon the improved ping module and hurdle based routing algorithm, where the proposed work has proved well in terms of dealy (or latency). The latency is the parameter, which signifies the detection delay produced by the ping packets in the particular point of time in accordance with the best time taken for the route hurdle discovery and information updation. The latency has been improved in the proposed system at the rate of approx $15 \%$.

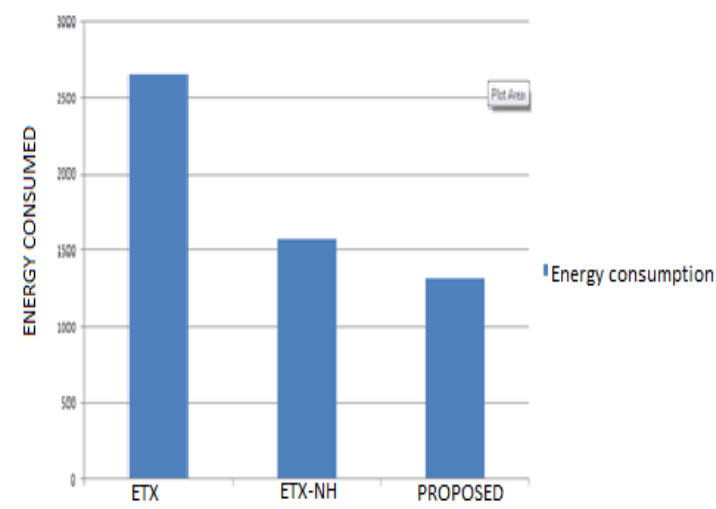

Fig 2: The graph of energy consumption
The proposed model have proved the efficiency of the proposed model in energy overhead caused due to the packet transmission. The packet transmission if will be less due to any process, it will directly affect the energy consumption of the proposed model. The energy consumption improvement in the case of proposed model has been recorded with improvement of almost $15 \%$.

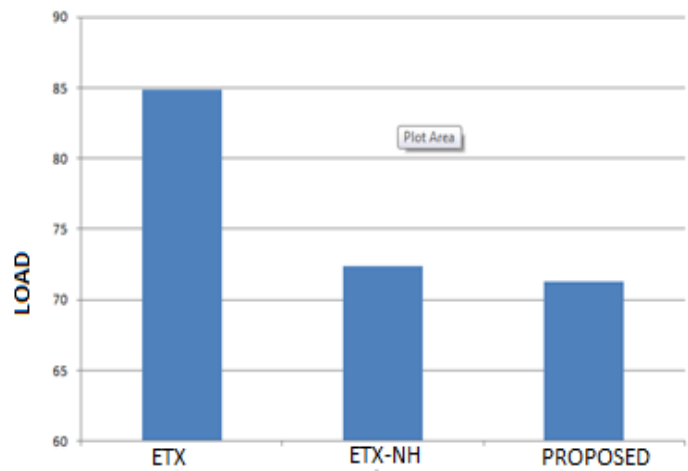

Fig 3: The comparative analysis of load in ping module

The ping load is the indicator of the data volume on the ping module. The higher ping load is produced by receiving the multiple ping replies altogether. The load can be decreased by using an effective method to compute the duplicate points of hurdles replies from the same hurdle on different points. Such unique point evaluation can be utilized for the unique and still object evaluation for the purpose of unique hurdle point evaluation. The ping load is the load indicator, which signifies the use of the resources on a point of time. The ping load varies due to the height of the flight and geographical area. The improvement in the case of load has been recorded at almost $10 \%$ from the existing system.

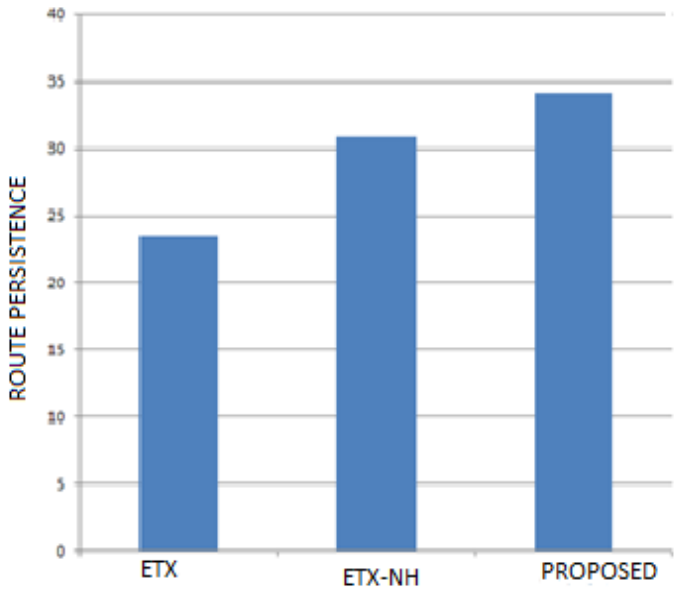

Fig 4: The route persistence comparison

The route persistence is the parameters, which signifies the elongation of the available routes. It means the availability of the route for the elongated period. The route persistence is higher in the case of proposed model than the existing model. The improvement in the route persistence has been recorded with the improvement more than $20 \%$.

\section{CONCLUSION}

The UAV routing module has been designed with the amalgamation of the active hurdle detection module using the ping discovery mechanism along with the path information 
update algorithm, which utilizes the angle of rotation or elevation angle for the flight path updation across the path from the source to the destination. The proposed model has been designed with the active fuel tracking algorithm along with the active hurdle evaluation for the uninterrupted flight path in the adverse conditions, such as hilly areas, high density skyscrapers in the urban areas, etc. The proposed model has been evaluated for its performance in the terms of route stability calculated using the route persistence. Also the ping load has been evaluated to measure the ratio of the processing capacity to the number of ping replies available for processing the hurdles and updating the path information. The proposed model has been improved by almost $20 \%$ when evaluated on the basis of route persistence, $10 \%$ over the ping load and $15 \%$ lower path information update delay.

\section{REFERENCES}

[1] Adil Mudasir Malla and Ravi Kant Sahu, "Security Attacks with an Effective Solution for DOS Attacks in VANET", in International Journal of Computer Applications, March 2013, Volume 66 - Number 22.

[2] B. Parno and A. Perrig, "Challenges in Securing Vehicular Networks", in Hot Topics in Networks (HotNets-IV), 2005.

[3] Bachar Wehbi, Edgardo Montes de Oca, Michel Bourdelles, "Events- Based Security Monitoring Using MMT Tool ", in Software Testing, Verification, and Validation, 2008 International Conference, 2012, pp. 860-863. International Journal on AdHoc Networking Systems (IJANS) Vol. 4, No. 2, April 2014

[4] Bin Xiao, Bo Yu, Chuanshan Gao, "Detection and localization of Sybil nodes in VANETs", in DIWANS '06, pp. 1-8.
[5] Chenxi Zhang, Rongxing Lu, Xiaodong Lin, Pin-Han Ho, and Xuemin (Sherman) Shen, "An Efficient IdentityBased Batch Verification Scheme for Vehicular Sensor Networks", in IEEE INFOCOM 2008 proceedings, 2008, pp. 816-824.

[6] Chia-Chen Hung, Hope Chan, and Eric Hsiao-Kuang Wu "Mobility Pattern Aware Routing for Heterogeneous Vehicular Networks"( IEEE WCNC 2008).

[7] Collins, Gaemus E., James R. Riehl, and Philip S. Vegdahl. "A UAV routing and sensor control optimization algorithm for target search." In Defense and Security Symposium, pp. 65610D-65610D. International Society for Optics and Photonics, 2007.

[8] .Sundar, Kaarthik, and Sivakumar Rathinam. "Algorithms for routing an unmanned aerial vehicle in the presence of refueling depots." Automation Science and Engineering, IEEE Transactions on 11.1 (2014): 287-294.

[9] .Manyam, Satyanarayana G., et al. "Routing of two Unmanned Aerial Vehicles with communication constraints." Unmanned Aircraft Systems (ICUAS), 2014 International Conference on. IEEE, 2014.

[10] .Hernandez-Hernandez, Lucia, et al. "Multi-Objective UAV routing." Unmanned Aircraft Systems (ICUAS), 2014 International Conference on. IEEE, 2014.

[11] Klein, Daniel J., et al. "On UAV routing protocols for sparse sensor data exfiltration." American Control Conference (ACC). 2010 